\title{
APPROXIMATION OF A SEMILINEAR ELLIPTIC PROBLEM IN AN UNBOUNDED DOMAIN
}

\author{
Messaoud Kolli $^{1}$ and Michelle Schatzman ${ }^{2}$
}

\begin{abstract}
Let $f$ be an odd function of a class $\mathrm{C}^{2}$ such that $f(1)=0, f^{\prime}(0)<0, f^{\prime}(1)>0$ and $x \mapsto f(x) / x$ increases on $[0,1]$. We approximate the positive solution of $-\Delta u+f(u)=0$, on $\mathbb{R}_{+}^{2}$ with homogeneous Dirichlet boundary conditions by the solution of $-\Delta u_{L}+f\left(u_{L}\right)=0$, on $] 0, L\left[^{2}\right.$ with adequate non-homogeneous Dirichlet conditions. We show that the error $u_{L}-u$ tends to zero exponentially fast, in the uniform norm.
\end{abstract}

Mathematics Subject Classification. 35J60, 35P15.

Received: August 30, 2001. Revised: September 30, 2002.

\section{INTRODUCTION}

Let $f$ be a function from $[-1,1]$ to $\mathbb{R}$, which satisfies the following assumptions:

$f$ is twice continuously differentiable;

$f$ is odd, $f(1)=0, f^{\prime}(0)<0, f^{\prime}(1)>0$;

the function $x \mapsto f(x) / x$ is strictly increasing over $[0,1]$.

In this article, we consider the semilinear equation

$$
\begin{aligned}
& -\Delta u+f(u)=0, \text { in } \mathbb{R}^{2}, \\
& -1 \leq u \leq+1, \\
& \operatorname{sign}(u(x, y))=\operatorname{sign}(x y),
\end{aligned}
$$

and we study the approximation of the solution of (4) by problems on finite domains.

This problem has its origin in Allen and Cahn's time dependent model, which can be written

$$
u_{t}-\varepsilon^{2} \Delta u+f(u)=0
$$

Keywords and phrases. Semilinear elliptic equations, full-space problems, approximation by finite domains.

1 Département de Mathématiques, Université Ferhat-Abbas, Sétif 19000, Algérie. e-mail: kollimes@yahoo.fr

2 MAPLY, CNRS, Université Claude Bernard - Lyon 1, 21 Avenue Claude Bernard, 69622 Villeurbanne Cedex, France. e-mail: schatz@maply.univ-lyon1.fr 
this equation has been proposed as a description of boundary motion in alloys [1]. The parameter $\varepsilon$ is often taken small, and one of the essential features of (5) is that after a time $O(\ln (1 / \varepsilon))$, the value of the solution is either close to 1 or close to -1 , except on thin regions of width $O(\varepsilon)$; see [7] or [3] for an exposition of the generation of interfacial regions. After interfaces are generated, they move and their motion is asymptotic to motion by mean curvature on a time scale $O\left(\varepsilon^{-2}\right)$; see [8] or [3] for the smooth case.

Loss of regularity occurs quite often for motion by mean curvature, and a number of mathematical devices have been invented to extend solutions beyond the onset of singularities; a Hamilton-Jacobi approach has been described in [10]; a geometric measure theory solution has been given in [14].

The simplest kind of singular situation in the plane is the case when the initial data for (5) has the sign structure given by (4c). If we blow up the spatial variable, we see that the sign condition (4c) is a rather natural requirement in the neighborhood of the simplest singularities of a two-dimensional solution.

Since we consider only the local structure, the scale becomes irrelevant, and we may assume that it is equal to 1 . In order to understand the evolution of a solution having the sign structure (4c), we first need to understand the unstable equilibria, i.e. the solutions of (4).

The existence and uniqueness of the non trivial solution of (4) has been proved by Fife et al. in [5]; this solution is twice continuously differentiable and satisfies the symmetry conditions

$$
\forall(x, y) \in \mathbb{R}^{2}, \quad u(x, y)=-u(-x, y)=-u(x,-y)
$$

Moreover, $u$ takes its values in the open interval $(-1,1)$ and is strictly positive on $(0, \infty) \times(0, \infty)$. Much asymptotic information on this solution has been obtained in [18].

But if we want more concrete knowledge about the solution of (4), we need to approximate it numerically. Since (4) is set in an unbounded domain, we have to approximate it by a solution on a bounded domain, after defining appropriate boundary conditions.

In the realm of fluid mechanics and problems dominated by propagation, many authors have devised artificial boundary conditions which enabled them to approximate in a finite domain something that takes place in an infinite domain; in the case of linear problems with propagation properties, the seminal paper of Engquist and Majda [9] paved the way for many further developments which are essentially unrelated to the present problem.

Before mentioning some results on artificial boundary conditions for elliptic equations, let us mention for ordinary differential equations the work of de Hoog and Weiss [6], of Jepson and Keller [15], see also Jepson's thesis [16], and of Markowich [17].

Very few authors have treated absorbing boundary conditions for elliptic operators; since our problem is non linear, the approximation method narrowly depends on the qualitative properties of the solution.

In our case, we are dealing with a second order operator, and we must think of the situation at infinity as extremely hyperbolic, where hyperbolicity must be understood in the sense of dynamical systems. More precisely, over odd functions, the linearized operator at the solution as $y$ tends to infinity is of the form $\partial^{2} / \partial y^{2}-A$, where $A$ is a positive elliptic operator in dimension 1 whose coefficients depend on $x$. Therefore it has a positive square root, and the operator can be factored as $(\partial / \partial y-\sqrt{A})(\partial / \partial y+\sqrt{A})$. The first factor corresponds to a well posed problem in the direction of decreasing $y$ and the second factor corresponds to a well-posed problem in the direction of increasing $y$.

We can exploit this dichotomy by obtaining a stable manifold behavior at infinity, and it is this feature which makes the construction of appropriate boundary condition possible.

In this respect, our philosophy is essentially the same as that of Hagstrom and Keller [12] and [13]; of course, none of their conditions holds in our case, and thus, we have to do quite a bit of analysis to reach the conclusion. However, the prize is well worth the effort, since we obtain finally that the error tends to 0 exponentially fast in uniform norm as the size of the domain tends to infinity.

We need more detailed information about the asymptotics obtained in [18]. The proof that Fife et al.'s solution is linearly unstable was based on a very precise study of the behavior at infinity of the solution; let 
indeed $\Theta$ denote the unique odd bounded and increasing non trivial solution of

$$
-\Theta^{\prime \prime}(x)+f(\Theta(x))=0, \quad x \in \mathbb{R} .
$$

This function $\Theta$ converges exponentially fast to its limits \pm 1 at $\pm \infty$; the existence of $\Theta$ is a straightforward application of the maximum principle, $\Theta$ is of class $\mathrm{C}^{4}$; as $(1,0)$ and $(-1,0)$ are hyperbolic points for the associated system of first order equations, we may apply the stable and unstable manifold theory described for instance in [4] (Chap. 3, Th. 8.1); the absolute value of the eigenvalues of the linearized problem is

$$
\beta=\sqrt{f^{\prime}(1)},
$$

so that the following estimates hold:

$$
\forall x \in \mathbb{R}, \quad \sum_{k=0}^{4}\left|\frac{\partial^{k}}{\partial x^{k}}(1-\Theta(x))\right| \leq C \mathrm{e}^{-\beta|x|} .
$$

It has been proved in [18], that there exist two strictly positive constants $\mu$ and $C_{\mu}$ such that

$$
\forall(x, y) \in \mathbb{R}^{2}, \quad \sum_{\alpha_{1}+\alpha_{2} \leq 3} \sup _{(x, y) \in \mathbb{R}^{2}}\left|\frac{\partial^{\alpha_{1}}}{\partial x^{\alpha_{1}}} \frac{\partial^{\alpha_{2}}}{\partial y^{\alpha_{2}}}(u(x, y)-\Theta(x))\right| \leq C_{\mu} \mathrm{e}^{-\mu|y|} .
$$

The symmetric condition holds when $x$ and $y$ are exchanged.

In this article, we show that it is possible to approximate the solution of (4) by solving a problem on the square

$$
\Omega_{L}=(0, L) \times(0, L)
$$

with appropriate boundary conditions.

First, the symmetry conditions (6) enable us to work in a quarter-plane; we show in Section 1 that the problem

$$
\begin{aligned}
& -\Theta_{L}^{\prime \prime}+f\left(\Theta_{L}\right)=0, \text { in the sense of distributions on }(0, L) \\
& \Theta(0)=0, \quad \Theta(L)=1 \\
& 0 \leq \Theta \leq 1
\end{aligned}
$$

possesses a unique solution. Our approximation of the solution of (4) uses a boundary condition defined with the help of a function $g_{L}$ given by

$$
g_{L}(x, y)=\Theta_{L}(x) \Theta_{L}(y) .
$$

The two dimensional problem will then be

$$
\begin{aligned}
& -\Delta u_{L}+f\left(u_{L}\right)=0, \text { in the sense of distributions on } \Omega_{L}, \\
& \left.\left(u_{L}-g\right)\right|_{\partial \Omega_{L}}=0, \\
& 0 \leq u_{L} \leq 1 .
\end{aligned}
$$

Let us outline now the organization of this article. In Section 2, we show that (13) possesses a unique solution for all strictly positive $L$; in Section 3 we show that the sequence of solutions $\left(u_{L}\right)$ converges in an appropriate sense to $u$, solution of (4), as $L$ tends to infinity; in both sections we use repeatedly the maximum principle. We 
show first the uniform convergence using the maximum principle; then we refine the spectral study performed in [5], to show a spectral result which builds on results from that article. More precisely, let $B$ be the linearized operator of (4) at $u$; call $B_{\sigma}$ the restriction of $B$ to the space of functions possessing the symmetry property (6); then the lower bound of the spectrum of $B_{\sigma}$ is strictly positive. This information enables us to conclude that the mean square norm of the error is exponentially small; this estimate, together with elliptic estimates shows the exponentially fast convergence of the maximum norm as $L$ tends to infinity.

\section{The ONE-DIMENSIONAL PROBLEM}

Let us start by an easy regularity result:

Lemma 1.1. Let $v$ be a measurable function on $(0, L)$ taking its values in $[0,1]$ and satisfying

$$
-v^{\prime \prime}+f(v)=0 \text {, in the sense of distributions. }
$$

Then $v$ is of class $\mathrm{C}^{4}$.

Proof. Relation (14) implies that $v^{\prime \prime}$ is essentially bounded; therefore, $v^{\prime}$ is Lipschitz continuous over [0,L], $v$ is of class $\mathrm{C}^{1}$, and $f(v)$ is of class $\mathrm{C}^{1}$; but then, $v$ is of class $\mathrm{C}^{3}, f(v)$ is of class $\mathrm{C}^{2}$ and the result is proved.

Let us start by proving the uniqueness of the solutions to (11):

Lemma 1.2. Problem (11) has at most one solution.

Proof. We argue by contradiction. Assume that there exist two solutions $v_{1}$ and $v_{2}$; since they are continuous and they satisfy the same Dirichlet conditions,

$$
U=\left\{x \in[0, L]: v_{1}(x) \neq v_{2}(x)\right\}
$$

is an open subset of $(0, L)$; let $(a, b)$ be a connected component of $U$; without loss of generality,

$$
v_{1}>v_{2} \text { over }(a, b)
$$

we multiply respectively the equation for $v_{1}$ by $v_{2}$ and the equation for $v_{2}$ by $v_{1}$, we subtract the second from the first, and we integrate over $(a, b)$ :

$$
\int_{a}^{b}\left(-v_{1}^{\prime \prime} v_{2}+v_{2}^{\prime \prime} v_{1}\right) \mathrm{d} t+\int_{a}^{b}\left(\frac{f\left(v_{1}\right)}{v_{1}}-\frac{f\left(v_{2}\right)}{v_{2}}\right) v_{1} v_{2} \mathrm{~d} t=0 .
$$

By integration by parts the first integral in (17) is equal to

$$
-v_{1}^{\prime}(b) v_{2}(b)+v_{1}^{\prime}(a) v_{2}(a)+v_{2}^{\prime}(b) v_{1}(b)-v_{2}^{\prime}(a) v_{1}(a)
$$

By definition of $(a, b), v_{1}$ and $v_{2}$ coincide at the end points of that interval. But the inequality condition (17) implies that $v_{1}^{\prime}(b) \leq v_{2}^{\prime}(b)$ and similarly $v_{2}^{\prime}(a) \leq v_{1}^{\prime}(a)$; therefore, the expression (18) is non negative; in virtue of assumption (3), the second integral in (17) is strictly positive, and we have a contradiction.

We build $\Theta_{L}$ by a supersolution algorithm; recall the classical definition:

Definition 1.3. A function $z$ whose derivative is Lipschitz continuous is a supersolution for (11) if it satisfies the following inequalities

$$
\left\{\begin{array}{l}
-z^{\prime \prime}+f(z) \geq 0, \text { in the sense of measures } \\
\text { and almost everywhere on }(0,1), \quad z(0) \geq 0, \quad z(1) \geq 1 .
\end{array}\right.
$$

It is a subsolution if all the inequalities in (19) are reversed. 
Let $\kappa$ be a positive constant satisfying the following inequality:

$$
\kappa \geq \sup _{x \in[0,1]} f^{\prime}(x)
$$

We define a sequence of functions $\left(\Theta_{j}\right)_{j}$ inductively by

$$
\Theta_{0} \equiv 1
$$

and for $j \geq 0, \Theta_{j+1}$ is the unique solution of

$$
\begin{aligned}
& \left.-\Theta_{j+1}^{\prime \prime}(x)+\kappa \Theta_{j+1}(x)=\kappa \Theta_{j}(x)-f\left(\Theta_{j}(x)\right), \quad x \in\right] 0, L[, \\
& \Theta_{j+1}(0)=0, \quad \Theta_{j+1}(L)=1 .
\end{aligned}
$$

Lemma 1.4. The sequence $\left(\Theta_{j}\right)_{j \geq 0}$ has the following properties:

(i) For all $j \geq 1, \Theta_{j}$ takes its values in $[0,1]$ and more precisely

$$
\forall x \in(0, L), \quad 0<\Theta_{j}(x)<1 .
$$

(ii) The sequence $\left(\Theta_{j}\right)_{j \geq 0}$ is strictly decreasing:

$$
\forall x \in(0, L), \quad \forall j \geq 0, \quad \Theta_{j+1}(x)<\Theta_{j}(x) .
$$

(iii) For all $j \geq 0, \Theta_{j}$ is estimated from below by $\Theta$ :

$$
\forall x \in(0, L), \quad \Theta(x)<\Theta_{j}(x)
$$

(iv) If $\Theta_{L}$ is a solution of (11), then it is estimated from above by $\Theta_{j}$ :

$$
\forall j \geq 0, \quad \forall x \in] 0, L\left[, \quad \Theta_{j}(x)>\Theta_{L}(x) .\right.
$$

Proof. We use several times the strong maximum principle, which can be found for instance in Brezis' course book [2] (Cor. IX. 37) or in the classical book of Gilbarg and Trudinger [11].

(i) For $j=1, \Theta_{1}$ is the unique solution of $-\Theta_{1}^{\prime \prime}+\kappa \Theta_{1}=\kappa$; since one of the boundary values of $\Theta_{1}$ is strictly positive, the strong maximum principle implies that $\Theta_{1}$ is strictly positive over $(0, L)$; as $1-\Theta_{1}$ satisfies the equation $-\left(1-\Theta_{1}\right)^{\prime \prime}+\kappa\left(1-\Theta_{1}\right)$, the strong maximum principle implies that $1-\Theta_{1}$ is also strictly positive over $(0, L)$, and $(22)$ holds for $j=1$. Assume now that (22) is true for some index $j$; then, by definition of $\kappa$, the right-hand side of $(21 \mathrm{~b})$ is well defined and non negative. By the strong maximum principle, $\Theta_{j+1}$ and $1-\Theta_{j+1}$ are strictly positive over $(0, L)$.

(ii) According to (i), $\Theta_{1}<\Theta_{0}$ over $(0, L)$. If for some index $j, \Theta_{j}<\Theta_{j-1}$, we subtract equation (21b) for $\Theta_{j}$ from equation (21b) for $\Theta_{j+1}$ and we find that the difference $z=\Theta_{j+1}-\Theta_{j}$ satisfies

$$
-z^{\prime \prime}+\kappa z=\kappa\left(\Theta_{j-1}-\Theta_{j}\right)+f\left(\Theta_{j}\right)-f\left(\Theta_{j-1}\right) \text { over }(0, L)
$$

the right-hand side of this equation is non negative, and therefore, by the strong maximum principle, $z$ is strictly positive over $(0, L)$, proving thus (ii). 
(iii) Inequality (24) holds for $j=0$. If it is true for a certain index $j$, then $z=\Theta_{j+1}-\Theta$ is positive at the boundary of $(0, L)$ and it satisfies

$$
-z^{\prime \prime}+\kappa z=\kappa\left(\Theta_{j}-\Theta\right)+f(\Theta)-f\left(\Theta_{j}\right) \text { over }(0, L)
$$

as the right-hand side of the above equation is non negative, the strong maximum principle gives the conclusion.

(iv) The proof is completely analogous to that of (iii), and left to the reader.

We can conclude now the existence proof:

Theorem 1.5. The sequence of functions $\Theta_{j}$ converges in $\mathrm{C}^{2}([0, L])$ to the unique solution $\Theta_{L}$ of $(11)$.

Proof. For all $x \in[0, L]$, the sequence $\left(\Theta_{j}(x)\right)_{j \geq 0}$ is decreasing and non negative; therefore, it converges, and its limit is denoted by $\Theta_{L}$. Therefore, the sequence $\left(f\left(\Theta_{j}\right)\right)_{j \geq 0}$ converges everywhere and in $L^{1}$ to $f\left(\Theta_{L}\right)$ as $j$ tends to infinity; we can pass to the limit in the sense of distributions in (21b); thanks to Lemma $1.1, \Theta_{L}$ is a classical solution. The uniqueness has been proved at Lemma 1.2.

Remark 1.6. The convergence of the sequence $\Theta_{j}$ is uniform: relation (21b) gives a uniform bound on the second derivative of $\Theta_{j}$, and Ascoli-Arzela's theorem applies.

We summarize some properties of the solution $\Theta_{L}$ in next statement:

Theorem 1.7. The function $\Theta_{L}$ has the following properties:

$$
\begin{aligned}
& \Theta_{L} \text { is strictly increasing over }(0, L) \text {; } \\
& \Theta_{L}>\Theta \text { over }(0, L) \text {; } \\
& \text { If } \left.v \neq \Theta_{L} \text { is a sub(super }\right) \text { solution, then } v<\Theta_{L}\left(\text { respectively } v>\Theta_{L}\right) \text {. }
\end{aligned}
$$

Proof. As $x \mapsto f(x) / x$ is strictly increasing over [0,1], and $f(1)$ vanishes, $f$ is negative over $(0,1)$; therefore, $\Theta_{L}$ is a strictly concave function over $(0, L)$ which is at most equal to 1 ; if it attained a maximum at $x_{0} \in(0, L)$, this maximum would be equal to 1 and $\Theta_{L}$ would be identically equal to 1 over $\left[x_{0}, L\right]$, contradicting the strict concavity and proving thus $(26)$.

We infer from (24) that $\Theta \leq \Theta_{L}$ over $[0, L]$; but the difference $z=\Theta_{L}-\Theta$ verifies

$$
-z^{\prime \prime}+\kappa z=\kappa z+f(\Theta)-f\left(\Theta_{L}\right)
$$

whose right-hand side is non negative; as $z(L)$ is strictly positive, the strong maximum principle enables us to conclude that (27) holds.

Finally, if $v \neq \Theta_{L}$ is a supersolution, we put $\Theta_{0}=v$ in algorithm (21), and we use the uniqueness of the solution, proving thus (28); if $v$ is a subsolution, the argument can be modified appropriately.

\section{The BIDIMEnsional PROBLEM}

The construction of a solution of (13) is completely parallel to the construction of a solution of (11). We start with a regularity result:

Lemma 2.1. Let $v$ be a measurable solution of $(13)$; then $u$ belongs to $\mathrm{C}^{1, \alpha}\left(\overline{\Omega_{L}}\right)$.

Proof. The function $g_{L}$ defined by (12) is of class $\mathrm{C}^{4}$; the difference $w=v-g_{L}$ satisfies the equation

$$
\begin{aligned}
& -\Delta w=f(v)+\Delta g_{L}, \text { over } \Omega_{L}, \\
& \left.w\right|_{\partial \Omega_{L}}=0 \text { on } \partial \Omega_{L} ;
\end{aligned}
$$


the right-hand side $\phi=f(v)+\Delta g_{L}$ of (29) belongs to $L^{\infty}\left(\Omega_{L}\right)$; we extend $v$ as a periodic functions over all of $\mathbb{R}^{2}$, according to the following symmetry conditions

$$
\forall(x, y) \in \mathbb{R}^{2}: \quad\left\{\begin{aligned}
v(2 L-x, y)=-v(x, y), & v(x, 2 L-y)=-v(x, y), \\
v(x+2 L, y)=v(x, y), & v(x, y+2 L)=v(x, y) .
\end{aligned}\right.
$$

With a similar extension for $\phi$, equation (29) holds over all of $\mathbb{R}^{2}$, and thanks to the classical Agmon-DouglisNirenberg estimates, $v$ belongs to the Sobolev space $W_{\text {loc }}^{2, p}\left(\mathbb{R}^{2}\right)$ and therefore to the Hölder space $\mathrm{C}_{\text {loc }}^{1, \alpha}\left(\mathbb{R}^{2}\right)$; the conclusion is clear.

Theorem 2.2. Problem (13) has at least one solution, which is constructed by a supersolution algorithm similar to (21). Moreover, the solution constructed in this fashion is the maximal solution of (13).

Proof. For the existence, we define a sequence of functions $\left(u_{j}\right)_{j}$ by the algorithm

$$
u_{0} \equiv 1
$$

for $j \geq 0, u_{j+1}$ is the unique solution of

$$
\begin{aligned}
& -\Delta u_{j+1}+\kappa u_{j+1}=\kappa u_{j}-f\left(u_{j}\right), \text { in } \Omega_{L} \\
& \left.\left(u_{j+1}-g_{L}\right)\right|_{\partial \Omega_{L}}(x, y)=0 .
\end{aligned}
$$

Lemma 1.4 still holds if we replace $\Theta_{j}$ by $u_{j}, \Theta$ by $u, \Theta_{L}$ by $u_{L}$ and the interval $(0, L)$ by the square $\Omega_{L}$. Letting

$$
u_{L}(x, y)=\lim _{j \mapsto+\infty} u_{j}(x, y), \quad \forall(x, y) \in \bar{\Omega}_{L},
$$

the reader will check that, as in the one-dimensional case, $u_{L}$ is a measurable solution of (13).

Let us prove now the uniqueness:

Theorem 2.3. The solution defined at Theorem 2.2 is the unique solution of (13).

Proof. Let $v$ be another solution of (13); we know from Lemma 2.1 that $v$ is of class $\mathrm{C}^{1, \alpha}$ for all $\alpha \in(0,1)$ and that it is at most equal to $u_{L}$; we multiply the equation for $v$ by $u_{L}$ and the equation for $u_{L}$ by $v$, we subtract the first equation from the second, we integrate over $\Omega_{L}$, we integrate by parts and we find the identity

$$
\int_{\partial \Omega_{L}} \frac{\partial\left(v-u_{L}\right)}{\partial \nu} g_{L} \mathrm{~d} \sigma+\int_{\Omega_{L}}\left(\frac{f\left(u_{L}\right)}{u_{L}}-\frac{f(v)}{v}\right) \mathrm{d} x \mathrm{~d} y=0 .
$$

Since $u_{L}$ is a maximal solution, the outer normal derivative of $v-u_{L}$, which is a classical derivative, thanks to Lemma 2.1, is non negative. The second integral in (33) is strictly positive as soon as $u_{L}$ is not identically equal to $v$; we obtain a contradiction.

Finally, we will need the following properties $u_{L}$ :

Theorem 2.4. The solution $u_{L}$ of (13) has the following properties:

$$
\begin{aligned}
& \text { If } \left.v \neq u_{L} \text { is a sub(super)solution, then } v<u_{L} \text { (respectively } v>u_{L}\right) \\
& \forall(x, y) \in \Omega_{L}, \quad u(x, y)<u_{L}(x, y)<\min \left(\Theta_{L}(x), \Theta_{L}(y)\right) .
\end{aligned}
$$


Proof. The proof of (34) is identical to the proof given in the one-dimensional case; details are left to the reader.

In [5], it is proved that the solution $u$ of (4) is estimated from above on $(0, \infty) \times(0, \infty)$ by $\min (\Theta(x), \Theta(y))$; as $\Theta<\Theta_{L}$ on $(0, L)$, the restriction of $u$ to $\Omega_{L}$ is a subsolution for problem (13), and property (34) implies the first inequality in (35). On the other hand, $(x, y) \mapsto \Theta_{L}(x)$ is a supersolution for (13) and we use (34) once again.

\section{Convergence as $L$ tends to infinity}

In this section we prove the exponentially fast convergence of $\Theta_{L}$ to $\Theta$ and hence the convergence of $u_{L}$ to $u$.

Lemma 3.1. Recall that $\beta$ is defined by (8). There exists a constant $C>0$ such that for all $L>0$ the following inequality holds:

$$
\max _{[0, L]}\left|\Theta_{L}(x)-\Theta(x)\right| \leq C \mathrm{e}^{-\beta L} .
$$

Proof. We multiply the two equations

$$
\begin{aligned}
& -\Theta_{L}^{\prime \prime}+f\left(\Theta_{L}\right)=0 \\
& -\Theta^{\prime \prime}+f(\Theta)=0
\end{aligned}
$$

respectively by $\Theta$ and $\Theta_{L}$, and for all $x \in[0, L]$, we integrate the difference over $[0, x]$; we obtain

$$
\int_{0}^{x}\left(\Theta^{\prime \prime} \Theta_{L}-\Theta \Theta_{L}^{\prime \prime}\right) \mathrm{d} t+\int_{0}^{x}\left(\frac{f\left(\Theta_{L}\right)}{\Theta_{L}}-\frac{f(\Theta)}{\Theta}\right) \Theta_{L} \Theta \mathrm{d} t=0 .
$$

The first integral in the above expression is equal to $\Theta^{\prime}(x) \Theta_{L}(x)-\Theta_{L}^{\prime}(x) \Theta(x)$ and the second one is strictly positive. Therefore, we have the inequality

$$
\forall x \in(0, L], \quad \Theta^{\prime}(x) \Theta_{L}(x)<\Theta_{L}^{\prime}(x) \Theta(x) .
$$

We infer from this inequality that the function $\ln \left(\Theta_{L} / \Theta\right)$ is strictly increasing, and therefore if $1-C_{\Theta} \mathrm{e}^{-\beta L}>0$, i.e. if $L$ is larger than some $L_{0}$,

$$
\max _{[0, L]} \frac{\Theta_{L}(x)}{\Theta(x)}=\frac{\Theta_{L}(1)}{\Theta(1)} \leq \frac{1}{1-C \mathrm{e}^{-\beta L}}
$$

We infer from (27) that

$$
\Theta<\Theta_{L}<\left(1-C \mathrm{e}^{-\beta L}\right)^{-1} .
$$

If $L \leq L_{0}$, we use the inequality $\left|\Theta-\Theta_{L}\right| \leq 1$, and the conclusion is clear.

Lemma 3.1 also implies estimates on the difference between the derivatives of $\Theta$ and those of $\Theta_{L}$ :

Corollary 3.2. There exists $C>0$ such that for all $L>0$ we have the estimate:

$$
\sum_{k \leq 4} \sup _{[0, L]}\left|D^{(k)}\left(\Theta_{L}-\Theta\right)(x)\right| \leq C \mathrm{e}^{-\beta L}
$$

Proof. If $v=\Theta_{L}-\Theta, v$ satisfies the ordinary differential equation

$$
v^{\prime \prime}=f\left(\Theta_{L}\right)-f(\Theta),
$$


with the boundary conditions

$$
v(0)=0, \quad v(L)=\Theta_{L}(L)-\Theta(L)
$$

Thanks to (36), we obtain immediately the estimate

$$
\max \left(\max _{[0, L]}\left|\Theta_{L}^{\prime}(x)-\Theta^{\prime}(x)\right|, \max _{[0, L]}\left|\Theta_{L}^{\prime \prime}(x)-\Theta^{\prime \prime}(x)\right|\right) \leq C \mathrm{e}^{-\beta L} .
$$

In order to obtain estimates on higher derivatives, we differentiate (40) twice, and with the help of (41) we get the desired conclusion.

Let us deal now with the convergence of $u_{L}$ to $u$; we introduce the notation $Q=(0, \infty) \times(0, \infty)$ for the open quarter-plane and we define extensions of $\Theta_{L}$ and $u_{L}$ respectively to $\mathbb{R}^{+}$and to $\bar{Q}$ :

$$
\tilde{\Theta}_{L}(x)= \begin{cases}\Theta_{L}(x) & \text { if } x \in[0, L] \\ 1 & \text { if } x \geq L\end{cases}
$$

and

$$
\tilde{u}_{L}(x, y)= \begin{cases}u_{L}(x, y) & \text { if }(x, y) \in \Omega_{L}, \\ \min \left\{\tilde{\Theta}_{L}(x), \tilde{\Theta}_{L}(y)\right\} & \text { otherwise. }\end{cases}
$$

Lemma 3.3. The sequence $\tilde{u}_{L}$ converges uniformly to $u$ as L tends to infinity.

Proof. The first step of the proof consists in checking that the sequences $\left(\tilde{\Theta}_{L}\right)_{L}$ and $\left(\tilde{u}_{L}\right)_{L}$ are decreasing with respect to $L$. Indeed, for $M>L$, the restriction of $\Theta_{M}$ to $(0, L)$ is a subsolution for (11) and the restriction of $u_{M}$ to $\Omega_{L}$ is a subsolution for (13); therefore, thanks to property (28), we have the inequality $\tilde{\Theta}_{M} \leq \tilde{\Theta}_{L}$ and thanks to property (34), we have also $\tilde{u}_{M} \leq \tilde{u}_{L}$; on the other hand, $\tilde{\Theta}_{L}$ is bounded from below by $\Theta$, and $\tilde{u}_{L}$ is bounded from below by $u$.

Therefore, the sequence $\tilde{u}_{L}$ has the point-wise limit $\tilde{u} \geq u$; it is plain that this convergence also holds in $L_{\text {loc }}^{p}(Q)$ and that in the limit (4a) holds on $Q$ with Dirichlet boundary conditions; moreover, $\tilde{u}$ takes its values in $[0,1]$. Together with this bound, the partial differential equation (4a) together with the bound implies that in fact $\tilde{u}$ belongs to $W_{\text {loc }}^{2, p}\left(\mathbb{R}^{2}\right)$ and hence is of class $\mathrm{C}^{1, \alpha}$ for all $\alpha \in(0,1)$; by bootstrapping, $\tilde{u}$ is of class $\mathrm{C}^{3, \alpha}$ for all $\alpha \in(0,1)$; by uniqueness of the solutions of (4), $\tilde{u}$ must be equal to $u$.

Let us partition now $Q$ into three regions, as depicted at Figure 1:

$$
\begin{aligned}
& R_{1}=[0, L) \times[L, \infty), \quad R_{2}=[L, \infty) \times[0, L), \\
& R_{3}=[L, \infty) \times[L, \infty), \quad R_{4}=[0, L) \times[0, L) .
\end{aligned}
$$

The function $u_{L}-u$ is given by:

$$
\tilde{u}_{L}-u= \begin{cases}\Theta_{L}(x)-u(x, y) & \text { on } R_{1} \\ \Theta_{L}(y)-u(x, y) & \text { on } R_{2} \\ 1-u(x, y) & \text { on } R_{3} \\ u_{L}(x, y)-u(x, y) & \text { on } R_{4}\end{cases}
$$

On $R_{1}$, we have the inequality

$$
\Theta_{L}(x)-u(x, y) \leq \Theta_{L}(x)-\Theta(x)+\Theta(x)-u(x, y)
$$




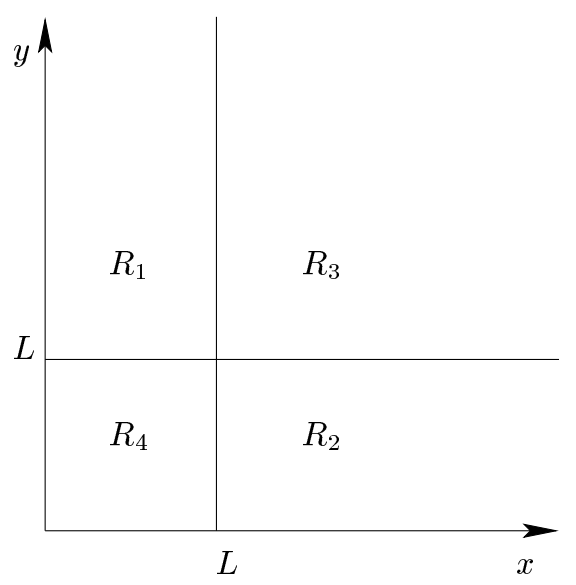

FiguRE 1 . The regions $R_{1}, R_{2}, R_{3}$ and $R_{4}$.

there is an analogous inequality in $R_{2}$; in $R_{3}$, we use directly estimate (10). If $\gamma$ denotes the minimum of $\beta$ and of the number $\mu$ appearing in (10), estimates (36) and (9) together with (10) imply that there exists a constant such that for all $L>0$,

$$
\forall(x, y) \in R_{1} \cup R_{2} \cup R_{3}, \quad 0 \leq \tilde{u}_{L}(x, y)-u(x, y) \leq C \mathrm{e}^{-\gamma L} .
$$

Given $\varepsilon$, we choose $L$ such that

$$
\sup _{R_{1} \cup R_{2} \cup R_{3}} \tilde{u}_{L}(x, y)-u(x, y) \leq \varepsilon
$$

then, for all $M \geq L$, we have also

$$
\sup \left\{\tilde{u}_{M}(x, y)-u(x, y):(x, y) \in R_{1} \cup R_{2} \cup R_{3}\right\} \leq \varepsilon .
$$

As the sequence $\tilde{u}_{M}$ decreases when $M$ tends to infinity to $\tilde{u}$, it converges uniformly to $u$ over $R_{1}$; therefore, we may choose $M$ so large that

$$
\sup _{R_{4}} u_{M}(x, y)-u(x, y) \leq \varepsilon
$$

thus we have proved the uniform convergence on $Q$ of $\tilde{u}_{L}$ to $u$.

However, we can prove now a much stronger result, namely that the sequence $u_{L}$ converges exponentially fast to $u$. This statement is is based on a result concerning the spectrum of the operator $B_{Q}$ defined by

$$
D\left(B_{Q}\right)=H^{2}(Q) \cap H_{0}^{1}(Q), \quad B_{Q} v=-\Delta v+f^{\prime}(u) v,
$$

and it is stated as a theorem:

Theorem 3.4. The lower bound of the spectrum of $B_{Q}$ is strictly positive. 


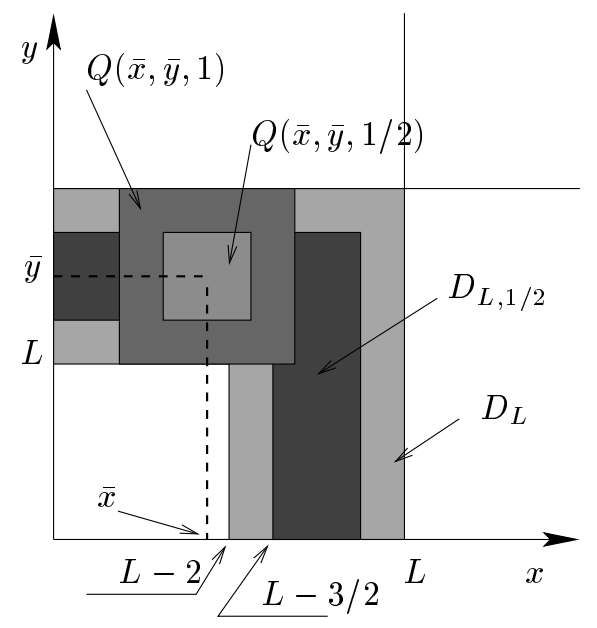

Figure 2. The regions $D_{L}, D_{L, 1 / 2}$ and the squares $Q(\bar{x}, \bar{y}, 1)$ and $Q(\bar{x}, \bar{y}, 1 / 2)$.

With this theorem, whose proof will be given at the end of the article, we can now show the following proposition:

Proposition 3.5. For all $\delta<\gamma$, there exists a constant $C$ such that for all $L>0$ :

$$
\sup _{\Omega_{L}} \max _{\alpha_{1}+\alpha_{2} \leq 2}\left|\frac{\partial^{\alpha_{1}+\alpha_{2}}\left(u_{L}(x, y)-u(x, y)\right)}{\partial x^{\alpha_{1}} \partial y^{\alpha_{2}}}\right| \leq C \mathrm{e}^{-\delta L} \text {. }
$$

Proof. It is an immediate consequence of Theorem 3.4 that there exists a number $\alpha>0$ such that

$$
\forall v \in H^{2}(Q) \cap H_{0}^{1}(Q), \quad\left(B_{Q} v, v\right) \geq \alpha\|v\|_{L^{2}(Q)}^{2} .
$$

It would be tempting to write the partial differential equation satisfied by $\tilde{u}_{L}$, subtract from it the equation satisfied by $u$ and hope for the best; however, this does not come so easily, since the Laplacian of $\tilde{u}_{L}$ has masses along the sets $[0, L] \times\{L\},\{L\} \times[0, L]$ and $\{(x, x): x \geq L\}$. Therefore, we have to modify $\tilde{u}_{L}$ into something smoother.

Assume $L \geq 2$ and let $D_{L}$ and $D_{L, 1 / 2}$ be the regions

$$
\begin{aligned}
D_{L} & =\{(x, y) \in \bar{Q}: L-2 \leq \max (x, y) \leq L\}, \\
D_{L, 1 / 2} & =\{(x, y) \in \bar{Q}: L-3 / 2 \leq \max (x, y) \leq L-1 / 2 .
\end{aligned}
$$

These regions are pictured in Figure 2. Estimates (10) and (35) imply that there exists a constant $C$ such that

$$
\forall(x, y) \in D_{L}, \quad 0 \leq u_{L}(x, y)-u(x, y) \leq C \mathrm{e}^{-\gamma L} .
$$

This implies that on $D_{L}$ we have the estimates

$$
\left|\Delta\left(u-u_{L}\right)\right| \leq C \mathrm{e}^{-\gamma L}
$$

Let now $Q(\bar{x}, \bar{y}, r)$ be the square centered at $(\bar{x}, \bar{y})$ with sides parallel to the axes and half side $r$; by interior Agmon-Douglis-Nirenberg estimates, for all $p \in[1, \infty)$, and all $r<1$ there exists a constant $C$ such that

$$
\|w\|_{W^{2, p}(Q(\bar{x}, \bar{y}, r))} \leq C\left(\|w\|_{L^{p}(Q(\bar{x}, \bar{y}, 1))}+\|w\|_{L^{p}(Q(\bar{x}, \bar{y}, 1))}\right)
$$


we choose $(\bar{x}, \bar{y})$ in $[0, L-1] \times\{L-1\}$ or in the symmetric set $\{L-1\} \times[0, L-1]$; then, by Sobolev injections, we see that there exists a constant $C$ such that for all $L \geq 2$,

$$
\sup _{1 \leq \bar{x} \leq L-1}\left\|u-u_{L}\right\|_{C^{1, \alpha}(Q(\bar{x}, L-1, r))} \leq C \mathrm{e}^{-\gamma L}
$$

if we bootstrap with interior Schauder estimates, we find that

$$
\sup _{1 \leq \bar{x} \leq L-1}\left\|u-u_{L}\right\|_{C^{2, \alpha}(Q(\bar{x}, L-1,1 / 2))} \leq C \mathrm{e}^{-\gamma L} .
$$

In order to deal with estimates close to $x=0$ or to $y=0$, we observe that this is a situation in which we may apply Agmon-Douglis-Nirenberg estimates up to the boundary; with details being left to the reader, we find eventually that there is a constant $C$ such that for all $L \geq 2$,

$$
\sup _{(x, y) \in D_{L, 1 / 2}} \max _{\alpha_{1}+\alpha_{2} \leq 2}\left|\frac{\partial^{\alpha_{1}+\alpha_{2}}\left(u-u_{L}\right)}{\partial x^{\alpha_{1}} \partial y^{\alpha_{2}}}\right| \leq C \mathrm{e}^{-\gamma L} .
$$

Let now $\omega$ be a non negative function of class $\mathrm{C}^{\infty}$ on $\mathbb{R}^{2}$ which is equal to 1 for $\max (x, y) \leq-1 / 4$ and to 0 for $\max (x, y) \geq 1 / 4$; we let

$$
\omega_{L}(x, y)=\omega(x-L+1, y-L+1)
$$

and we define

$$
\bar{u}_{L}=\tilde{u}_{L} \omega_{L}+u\left(1-\omega_{L}\right) .
$$

The function

$$
\rho_{L}=-\Delta \bar{u}_{L}+f\left(\bar{u}_{L}\right)
$$

is given by

$$
\rho_{L}=2 \nabla\left(u-\bar{u}_{L}\right) \cdot \nabla \omega_{L}+\left(u-\bar{u}_{L}\right) \Delta \omega_{L}+f\left(\bar{u}_{L} \omega_{L}+\left(1-\omega_{L}\right) u\right)-f\left(\bar{u}_{L}\right) \omega_{L}-f(u)\left(1-\omega_{L}\right) .
$$

We observe on expression (46) that the support of $\rho_{L}$ is included in $D_{L, 1 / 2}$, and we infer from estimates (45) that

$$
\left|\rho_{L}\right| \leq 1_{D(L, 1 / 2)} C \mathrm{e}^{-\gamma L} .
$$

Therefore, the difference $v_{L}=\bar{u}_{L}-u$ satisfies the equation

$$
-\Delta v_{L}+f^{\prime}(u) v_{L}=f^{\prime}(u) v_{L}-f\left(u+v_{L}\right)+f(u)+\rho_{L} .
$$

By construction, $v_{L}$ has compact support and therefore it belongs to $H^{2}(Q)$; it satisfies Dirichlet boundary conditions, and therefore, we may apply the spectral estimate (44): we multiply scalarly (47) by $v_{L}$, we integrate over $Q$ and we find

$$
\alpha\left\|v_{L}\right\|_{L^{2}(Q)}^{2} \leq\left\|v_{L}\right\|_{L^{2}(Q)}\left\|\rho_{L}\right\|_{L^{2}(Q)}+\left\|f^{\prime}(u) v_{L}-f\left(u+v_{L}\right)+f(u)\right\|_{L^{2}(Q)}\left\|v_{L}\right\|_{L^{2}(Q)} .
$$

As $f$ is of class $\mathrm{C}^{2}$, there exists a constant $C_{1}$ such that

$$
\left\|f^{\prime}(u) v_{L}-f\left(u+v_{L}\right)+f(u)\right\|_{L^{2}(Q)} \leq C_{1}\left\|v_{L}\right\|_{L^{\infty}(Q)}\left\|v_{L}\right\|_{L^{2}(Q)} .
$$


Therefore, we have obtained the estimation

$$
\left(\alpha-C_{1}\left\|v_{L}\right\|_{\mathrm{L}^{\infty}(Q)}\right)\left\|v_{L}\right\|_{\mathrm{L}^{2}(Q)} \leq\left\|v_{L}\right\|_{\mathrm{L}^{2}(Q)} .
$$

As $v_{L}$ is a convex combination of $u$ and $\tilde{u}_{L}$, we see that there exists $L_{0} \geq 2$ such that, for all $L \geq L_{0}$, $C_{1}\left\|v_{L}\right\|_{L^{\infty}(Q)}$ is less than or equal to $\alpha / 2$, and therefore, we have shown that there exists a constant $C$ such that for $L \geq L_{0}$

$$
\left\|v_{L}\right\|_{L^{2}(Q)} \leq C \sqrt{L} \mathrm{e}^{-\gamma L}
$$

but, going back to the partial differential equation satisfied by $v_{L}$, we infer immediately from (48) that

$$
\left\|v_{L}\right\|_{H^{2}(Q)} \leq C \sqrt{L} \mathrm{e}^{-\gamma L} .
$$

By the Sobolev injection of $H^{2}(Q)$ in $L^{\infty}(Q)$, we see that

$$
\left\|v_{L}\right\|_{L^{\infty}(Q)} \leq C \mathrm{e}^{-\gamma L} .
$$

Together with (45), this proves (43) for $\alpha_{1}=\alpha_{2}=0$; but we can apply again Agmon-Douglis-Nirenberg and Schauder estimates to the elliptic equation in $Q$

$$
-\Delta v_{L}=f(u)-f\left(u+v_{L}\right)
$$

with Dirichlet boundary conditions, and this implies (43) for $\alpha_{1}+\alpha_{2} \leq 2$, for $L \geq L_{0}$; for $L \leq L_{0}$, it is easy to fin an uniform bound on the derivatives of $u_{L}$ of order 1 and 2 , and the proposition is proved.

There remains to prove the spectral theorem which played such an essential rôle in the proof of Proposition 3.5.

Proof of Theorem 3.4. Let $B$ be the linearized operator at $u$ given by

$$
D(B)=H^{2}\left(\mathbb{R}^{2}\right), \quad B v=-\Delta v+f^{\prime}(u) v ;
$$

this operator is self-adjoint in $L^{2}\left(\mathbb{R}^{2}\right)$, and it has been proved in [18] that the essential spectrum of $B$ is included in $[0, \infty)$ and that if $\lambda$ is a negative eigenvalue of $B$, then the corresponding eigenfunction has the symmetries of the square.

Let $L^{2}\left(\mathbb{R}^{2}, \sigma\right)$ denote the subspace of functions of $L^{2}\left(\mathbb{R}^{2}\right)$ which have the symmetries $(6)$, understood in the almost everywhere sense. Thanks to the symmetry of $f^{\prime}(u), B$ maps a function of $L^{2}\left(\mathbb{R}^{2}, \sigma\right) \cap H^{2}\left(\mathbb{R}^{2}\right)$ to a function of $L^{2}\left(\mathbb{R}^{2}, \sigma\right)$. Therefore, there is a self adjoint operator $A_{\sigma}$ in $L^{2}\left(\mathbb{R}^{2}, \sigma\right)$ which is simply the restriction of $A$ to $D(A) \cap L^{2}\left(\mathbb{R}^{2}, \sigma\right)$.

The spectrum of $A_{\sigma}$ is included in $[0, \infty)$; indeed, the spectrum of $A_{\sigma}$ is included in the spectrum of $A$ and the essential spectrum of $A_{\sigma}$ is included in the essential spectrum of $A$, as can be seen on the characterization of the essential spectrum by singular sequences. Therefore, if the spectrum of $A_{\sigma}$ contained a negative number, it would be an eigenvalue, and the corresponding eigenfunction would have all the symmetries of the square, which contradicts the above mentioned result of [18].

Let us show now that the lower bound of the spectrum of $B_{\sigma}$ is strictly positive: we argue basically as in the proof of the analogous theorem in Lemmas 3.1 and 3.2 of [18] to which the reader is referred for all the necessary details. Let indeed $A$ be the operator defined by

$$
D(A)=H^{2}(\mathbb{R}), \quad A v=-v^{\prime \prime}+f^{\prime}(\Theta) v ;
$$


for this operator, the lower bound of the essential spectrum is $\beta^{2}$, and below that limit, the spectrum consists of simple eigenvalues

$$
\lambda_{0}<\lambda_{1}<\cdots<\beta^{2}
$$

the parity of the eigenfunction $v_{j}$ corresponding to $\lambda_{j}$ is the parity of $j, \lambda_{0}$ is equal to 0 , since the corresponding eigenfunction can be taken equal to $\Theta^{\prime}$; the corresponding operator on odd functions is called $A_{\sigma}$, defined by

$$
D\left(A_{\sigma}\right)=D(A) \cap\{\text { odd functions }\}, \quad A_{\sigma} u=A u ;
$$

it is immediate that the lower bound of the spectrum of $A_{\sigma}$ is equal to $\lambda_{1}$ if such an eigenvalue exists, and otherwise to $\beta^{2}$; it will be convenient to abuse notation and to write also $\lambda_{1}$ in the latter case.

Arguing as in [18], we see that the essential spectrum of $B_{\sigma}$ is identical with the essential spectrum of $A_{\sigma} \otimes \mathbf{1}+\mathbf{1} \otimes A_{\sigma}-\beta^{2}$, since the potential appearing in this Schrödinger operator has the same limit at infinity as the potential appearing in $B_{\sigma}$; therefore, the essential spectrum of $B_{\sigma}$ has the lower bound $\beta^{2}+\lambda_{1}-\beta^{2}=\lambda_{1}>0$.

There remains to show that 0 cannot be an eigenvalue of $B_{\sigma}$.

Assume therefore that $v$ belongs to the kernel of $B_{\sigma}$. We show first that it tends exponentially fast to 0 at infinity. For this purpose, we recall the definition of the operator $\mathrm{A}$ in $C_{b}^{0}$, the space of bounded continuous functions over $\mathbb{R}$; this operator was called $M$ in [18]:

$$
D(\mathrm{~A})=\mathrm{C}^{2}(\mathbb{R}) \cap\{\text { odd functions }\}, \quad \mathrm{A} z=-z^{\prime \prime}+f^{\prime}(\Theta) z ;
$$

it has been shown in [18] that there is a square root of $\mathrm{A}$ which generates an holomorphic semi-group whose norm decreases exponentially fast at infinity; more precisely, for all $\mu \in\left(0, \sqrt{\lambda_{1}}\right)$, there exists a constant $C_{\mu}$ such that

$$
\left\|\mathrm{e}^{-y \sqrt{\mathrm{A}}}\right\|_{\mathcal{L}\left(C_{b}^{0}\right)} \leq C_{\mu} \mathrm{e}^{-\mu y} .
$$

Our eigenfunction $v$ is bounded since it belongs to $H^{2}\left(\mathbb{R}^{2}\right)$.

We see that $v$ also solves the following Dirichlet problem in $\mathbb{R} \times[0, \infty)$ :

$$
-\Delta v+f^{\prime}(\Theta(x)) v=h, \quad v(x, 0)=0,
$$

with $h$ the function given by

$$
h(x, y)=\left(f^{\prime}(\Theta(x))-f^{\prime}(u(x, y))\right) v(x, y) .
$$

Therefore, according to Lemma 2.7 of [18], the solution of (50) is given by

$$
\begin{aligned}
v(\cdot, y)= & \mathrm{e}^{-y \sqrt{\mathrm{A}}}\left(v(\cdot, 0)+\frac{\mathrm{A}^{-1 / 2}}{2} \int_{0}^{\infty} \mathrm{e}^{-s \sqrt{\mathrm{A}}} h(\cdot, s) \mathrm{d} s\right) \\
& +\frac{\mathrm{A}^{-1 / 2}}{2} \int_{0}^{y} \mathrm{e}^{-(y-s) \sqrt{\mathrm{A}}} h(\cdot, s) \mathrm{d} s+\frac{\mathrm{A}^{-1 / 2}}{2} \int_{y}^{\infty} \mathrm{e}^{(y-s) \sqrt{\mathrm{A}}} h(\cdot, s) \mathrm{d} s .
\end{aligned}
$$

The asymptotic estimate (10) implies that that $h$ decays exponentially fast to 0 as $x$ tends to infinity, uniformly in $x$. Thus, the combination of (49) with formula (51) implies the exponential decay of $v$ to 0 at infinity; an 
application of Schauder and Agmon-Douglis-Nirenberg estimates enables us to conclude that

$$
\sup _{\alpha_{1}+\alpha_{2} \leq 2}\left|\frac{\partial^{\alpha_{1}+\alpha_{2}} v(x, y)}{\partial x^{\alpha_{1}} \partial y^{\alpha_{2}}}\right| \leq C \mathrm{e}^{-\nu y},
$$

for some $\nu \in(0, \mu)$.

There is of course an analogous estimate when $x$ tends to infinity.

As $v$ belongs to the kernel of $B_{\sigma}$, it also belongs to the kernel of $B_{Q}$, defined by (42); this means that the restriction of $v$ to $Q$ is a ground state for the operator $B_{Q}$, and in particular, it does not change sign.

We multiply the equation

$$
-\Delta v+f^{\prime}(u) v=0
$$

by $u$ and the equation (4a) by $v$, we subtract the second from the first, and we integrate over $Q$; as $u, v$ and their derivatives of order 1 tend to 0 exponentially fast at infinity, we integrate by parts safely, and we are left with the relation

$$
\int_{Q}\left(\frac{f(u)}{u}-f^{\prime}(u)\right) u v \mathrm{~d} x \mathrm{~d} y=0 .
$$

But assumption (3) implies that the factor in parentheses in the above integrand is of constant sign; therefore, $f(u)-u f^{\prime}(u)$ vanishes everywhere $Q$. As the relation

$$
f(z)-z f^{\prime}(z)=0
$$

is true for all $z \in[0, \sup u]$, on this interval, we must have $f(z)=C z$, which of course contradicts assumption (3). Thus, we have proved that the kernel of $B_{\sigma}$ is reduced to 0 , and Theorem 3.4 is proved.

The exponential convergence obtained in this article implies that the numerical approximation of the solutions of (4) can be safely performed in a bounded square; we can apply for that purpose any classical method: finite elements, finite differences, spectral methods; the numerical analysis of this problem will be presented in further work, together with the results of numerical simulations.

\section{REFERENCES}

[1] S. Allen and J. Cahn, A microscopic theory for antiphase boundary motion and its application to antiphase domain coarsening. Acta Metall. 27 (1979) 1084-1095.

[2] H. Brezis, Analyse fonctionnelle. Masson, Paris (1983). Théorie et applications [Theory and applications].

[3] Xinfu Chen, Generation and propagation of interfaces for reaction-diffusion equations. J. Differential Equations 96 (1992) $116-141$.

[4] E.A. Coddington and N. Levinson, Theory of ordinary differential equations. McGraw-Hill Book Company, Inc., New York, Toronto, London (1955).

[5] Ha Dang, P.C. Fife and L.A. Peletier, Saddle solutions of the bistable diffusion equation. Z. Angew. Math. Phys. 43 (1992) 984-998.

[6] F.R. de Hoog and R. Weiss, An approximation theory for boundary value problems on infinite intervals. Computing 24 (1980) $227-239$.

[7] P. de Mottoni and M. Schatzman, Development of interfaces in $\mathbb{R}^{N}$. Proc. Roy. Soc. Edinburgh Sect. A 116 (1990) $207-220$.

[8] P. de Mottoni and M. Schatzman, Geometrical evolution of developed interfaces. Trans. Amer. Math. Soc. 347 (1995) 15331589 .

[9] B. Engquist and A. Majda, Absorbing boundary conditions for the numerical simulation of waves. Math. Comp. 31 (1977) 629-651.

[10] L.C. Evans, H.M. Soner and P.E. Souganidis, Phase transitions and generalized motion by mean curvature. Comm. Pure Appl. Math. 45 (1992) 1097-1123.

[11] D. Gilbarg and N.S. Trudinger, Elliptic partial differential equations of second order. Springer-Verlag, Berlin (2001). Reprint of the 1998 edition. 
[12] T.M. Hagstrom and H.B. Keller, Asymptotic boundary conditions and numerical methods for nonlinear elliptic problems on unbounded domains. Math. Comp. 48 (1987) 449-470.

[13] T. Hagstrom and H.B. Keller, Exact boundary conditions at an artificial boundary for partial differential equations in cylinders. SIAM J. Math. Anal. 17 (1986) 322-341.

[14] T. Ilmanen, Convergence of the Allen-Cahn equation to Brakke's motion by mean curvature. J. Differential Geom. 38 (1993) $417-461$.

[15] A.D. Jepson and H.B. Keller, Steady state and periodic solution paths: their bifurcations and computations, in Numerical methods for bifurcation problems, Dortmund (1983). Birkhäuser, Basel (1984) 219-246.

[16] A. Jepson, Asymptotic boundary conditions for ordinary differential equations. Ph.D. thesis, California Institute of Technology (1980).

[17] P.A. Markowich, A theory for the approximation of solutions of boundary value problems on infinite intervals. SIAM J. Math. Anal. 13 (1982) 484-513.

[18] M. Schatzman, On the stability of the saddle solution of Allen-Cahn's equation. Proc. Roy. Soc. Edinburgh Sect. A 125 (1995) $1241-1275$.

To access this journal online:

www.edpsciences.org 